Noraiz Tahir - Francesco De Paolis • Asghar Qadir • Achille A. Nucita

\title{
Seeing the halo rotation of nearby spiral galaxies using Planck data
}

Received: 27 November 2018 / Accepted: 24 January 2019 / Published online: 7 February 2019

(C) The Author(s) 2019

\begin{abstract}
The rotation of the galactic halos is a fascinating topic which is still waiting to be addressed. Planck data have shown the existence of a temperature asymmetry towards the halo of several nearby galaxies, such as M31, NGC 5128, M33, M81 and M82. However, the cause of this asymmetry is an open problem. A possibility to explain the observed effect relies on the presence of "cold gas clouds" populating the galactic halos, which may be the answer to the so-called missing baryon problem. Here, we present a technique to estimate an upper limit to the rotational velocity of the halo of some nearby spiral galaxies using both their dynamical masses and the Planck data.
\end{abstract}

\section{Mathematics Subject Classification $\quad 85-00 \cdot 85-02 \cdot 85-05$}

\section{Introduction}

It is well known that baryons constitute about $5 \%$ of the Universe. Moreover, about half of them are missing, and it is not known in which form they are hidden. Different scenarios were proposed to address this issue (see, e.g., $[4,7,9,14,20,25])$. It was proposed that molecular hydrogen clouds at the cosmic microwave background (CMB) radiation temperature may contribute to the galactic halo dark matter [8]. If these clouds are at the CMB temperature, the question is how can they be detected. A suggestion was to look for a Doppler shift in the halo of the nearby Andromeda (M31) galaxy. The part of the halo rotating away from the observer will be red-shifted and the part rotating towards the observer will be blue-shifted. Planck data confirmed the effect for the M31 halo, and helped to study its rotational dynamics [10]. After that, Planck data allowed studying the dynamics of various other galaxies and their halos $[11,12,16,17]$. The composition, constituents and dynamics of the halo are obviously very difficult to determine. After all, by definition, this is the part of the galaxy that we cannot see but know of its presence due to the effect of its mass on the motion of the visible part. One can assume that "the halo rotates with the galaxy" but, as the galaxy does not rotate like a rigid disc, it would not be clear which part it "rotates with". There is need to be able to see the rotation of the halo. Due to the above effect, a new window is opened, to probe the dynamics of the halos of galaxies.

We know the temperature asymmetry is present, but the question is what causes this effect: it might be related to the presence of a hot, diffuse gas which emits in the CMB frequency region through Inverse

N. Tahir · A. Qadir

School of Natural Sciences, National University of Sciences and Technology, Islamabad, Pakistan

F. De Paolis · A. A. Nucita

Department of Mathematics and Physics "Ennio De Giorgi”, University of Salento, 73100 Lecce, Italy

F. De Paolis · A. A. Nucita ( $\square)$

INFN, Sezione di Lecce, CP 193, 73100 Lecce, Italy

E-mail: nucita@le.infn.it 
Compton scattering; to synchrotron emission by fast electrons; to anomalous microwave emission (AME) from dust grains; or to cold gas clouds populating the outer galaxy regions. Recently, a toy model was used for the cold gas clouds in the M31 halo using the isothermal Lane-Emden equation, provided that the density at the boundary of the cloud should merge with the density of the interstellar medium [26].

The aim of the present paper is to estimate, under the assumption that galactic halos are populated by cold gas clouds, the rotational velocity of some nearby galaxies using both their dynamical masses and the temperature asymmetry towards their halos detected in Planck data. The plan of the paper is as follows: In the next section, we will use three widely adopted models for the dark matter distribution in galactic halos to describe the distribution of the cold gas clouds. Then, in Sect. 3, we use both the galaxy dynamical mass and Planck data to derive an estimate of the halo rotation velocity of the NGC 5128, M33, M81, and M82 galaxies. In the last section, we offer our main conclusions.

\section{Dark matter distribution in galaxy halos}

The three most widely adopted models to describe the dark matter distribution in galaxy halos are those proposed by Navarro et al. [23], by Moore [22] and by Burkert [3]. The corresponding density distribution is given in the following equations

$$
\begin{aligned}
\rho_{\mathrm{NFW}}(r) & =\frac{\rho_{\mathrm{c}}}{\left[\frac{r}{r_{\mathrm{c}}}\right]\left[1+\frac{r}{r_{\mathrm{c}}}\right]^{2}}, \\
\rho_{\text {Moore }}(r) & =\frac{\rho_{\mathrm{c}}}{\left[\frac{r}{r_{\mathrm{c}}}\right]^{1.5}\left[1+\left(\frac{r}{r_{\mathrm{c}}}\right)^{1.5}\right]}, \\
\rho_{\text {Burkert }}(r) & =\frac{\rho_{\mathrm{c}}}{\left[1+\frac{r}{r_{\mathrm{c}}}\right]\left[1+\left(\frac{r}{r_{\mathrm{c}}}\right)^{2}\right]},
\end{aligned}
$$

where $\rho_{\mathrm{c}}$ is the central density and $r_{c}$ is the so-called core radius. The values of these parameters are given in Table 1 for the different considered galaxies, with the corresponding value of the dynamical mass [5,18,21,24]. We then assume that, as anticipated, cold gas clouds are distributed in the halos of the considered galaxies, following the mass distribution in Eqs. (1)-(3). Since the clouds are assumed to be isothermal and at the CMB temperature, we can use the Lane-Emden equation to obtain the mass density profile of each cloud. Now, a spherically symmetric cloud is described by the equation of hydrostatic equilibrium, i.e.,

$$
-\frac{1}{\rho(r)} \nabla P(r)-\nabla \phi(r)=0 .
$$

Here, $\rho(r)$ is the density of the cloud, $P(r)$ is the pressure applied by the molecules against the gravity, and $\phi(r)$ is the gravitational potential of the system. The equation of state for an ideal isothermal gas is

$$
P(r)=\rho(r) c_{\mathrm{s}}^{2},
$$

where $c_{\mathrm{S}}=\left(k T_{\mathrm{CMB}} / m_{\mathrm{H}}\right)^{1 / 2}$ is the sound speed, $k$ is the Boltzmann constant, $T_{\mathrm{CMB}} \approx 2.7254 \mathrm{~K}$, is the CMB temperature and $m_{\mathrm{H}} \approx 3.35 \times 10^{-25} \mathrm{~g}$ is the mass of single molecule of hydrogen. Now, the Poisson equation is

$$
\nabla^{2} \phi(r)=4 \pi G \rho(r)
$$

where $G$ is Newton's constant. From Eqs. (4) and (5) we have

$$
\rho(r)=\rho_{\mathrm{cl}} \exp (-\gamma),
$$

Equations (6) and (7) can be rewritten into the isothermal Lane-Emden equation, which is given by

$$
\frac{1}{\zeta^{2}} \frac{\mathrm{d}}{\mathrm{d} \zeta}\left(\zeta^{2} \frac{\mathrm{d} \gamma}{\mathrm{d} \zeta}\right)=\exp (-\gamma)
$$


where,

$$
\gamma=\frac{\phi}{c_{\mathrm{s}}^{2}}
$$

and

$$
\zeta=\sqrt{\frac{4 \pi G \rho_{\mathrm{cl}}}{c_{\mathrm{s}}^{2}}} r .
$$

This procedure was first proposed independently by Bonnor [2] and Ebert [13]. The Lane-Emden equation was solved, with the boundary condition that $\gamma(\zeta=0)=0$ and $\left.\frac{d \gamma}{d \zeta}\right|_{\zeta=0}=0$. The density distribution for an isothermal cloud is of the form

$$
\rho(r)=\rho_{\mathrm{cl}} e^{-\gamma} .
$$

Now the total mass of the cloud can be calculated as

$$
M_{\mathrm{cl}}=\int_{0}^{R_{\mathrm{cl}}} 4 \pi \rho(r) r^{2} \mathrm{~d} r
$$

where $\rho_{\mathrm{cl}}$ is the central density of the clouds and $\gamma=\phi / c_{s}$. It turns out that for our cold gas clouds, $c_{s} \simeq 3.3 \times 10^{4} \mathrm{~cm} \mathrm{~s}^{-1}$. The total mass of the cloud can be obtained by integrating the mass density distribution over the cloud up to $R_{\mathrm{cl}}$, using Eq. (12). Assuming that the cloud density at the boundary merges with that of the interstellar medium (IM). The mass $M_{\mathrm{cl}}$ and the radius $R_{\mathrm{cl}}$ of the clouds turn out to be $M_{\mathrm{cl}} \simeq 300 M_{\odot}$ and $R_{\mathrm{cl}} \simeq 56 \mathrm{pc}$. We note that the mass and the radius of the clouds are independent of the gas density at the boundary. In Table 1, we give the ratio of the central and the IM density for the galaxies, i.e., the values for $\rho_{c} / \rho_{\mathrm{IM}}$, where $\rho_{\mathrm{IM}}$ is the density of the IM. In Table 1 , the mass of each cloud, and the $\rho_{\mathrm{IM}}$, for each considered galaxy is shown. It is seen that the mass of the clouds turns out to be more or less the same for each of them. The central mass density of the gas clouds comes out to be $\rho_{\mathrm{cl}}=8.85 \times 10^{-21} \mathrm{gcm}^{-3}$.

Once we have the mass density, mass and the radius of the gas clouds, we can obtain the total number of clouds in the galactic halos of the considered galaxies. The total number of clouds in the galactic halo is given by the relation $N_{\text {total }}=f\left(M_{\text {Halo }} / M_{\mathrm{cl}}\right) . M_{\text {Halo }}$ is the total mass of the halo for each galaxy, $M_{\text {Halo }}(R)=\int_{0}^{R} 4 \pi r^{2} \rho_{\mathrm{N}, \mathrm{M}, \mathrm{B}}(r) \mathrm{d} r$. Here $R$ is the radius of the halo. The total number of gas clouds for each

\begin{tabular}{|c|c|c|c|c|c|c|}
\hline Galaxies & Models & $\begin{array}{l}\rho_{c} \\
\left(\mathrm{~g} \mathrm{~cm}^{-3}\right)\end{array}$ & $\begin{array}{l}r_{c} \\
(\mathrm{kpc})\end{array}$ & $n_{\mathrm{cl}} / n_{\mathrm{IM}}$ & $\begin{array}{l}M_{\mathrm{dyn}} \\
\left(10^{10} M_{\odot}\right)\end{array}$ & $\begin{array}{l}M_{\mathrm{cl}} \\
M_{\odot}\end{array}$ \\
\hline \multirow[t]{3}{*}{ Cen-A } & NFW & $1.61 \times 10^{-25}$ & 21 & $10^{5}$ & 40 & \multirow[t]{3}{*}{299.} \\
\hline & Moore & $4.40 \times 10^{-25}$ & 12 & $10^{5}$ & 40 & \\
\hline & Burkert & $1.0 \times 10^{-24}$ & 9.8 & $10^{5}$ & 40 & \\
\hline \multirow[t]{3}{*}{ M33 } & NFW & $7.0 \times 10^{-25}$ & 35.0 & $10^{5}$ & 80 & \multirow[t]{3}{*}{299.} \\
\hline & Moore & $7.64 \times 10^{-26}$ & 18.0 & $10^{5}$ & 80 & \\
\hline & Burkert & $5.0 \times 10^{-25}$ & 12.0 & $10^{5}$ & 80 & \\
\hline \multirow[t]{3}{*}{ M81 } & NFW & $5.10 \times 10^{-24}$ & 10 & $10^{6}$ & 200 & \multirow[t]{3}{*}{298.} \\
\hline & Moore & $7.65 \times 10^{-25}$ & 18 & $10^{6}$ & 200 & \\
\hline & Burkert & $3.68 \times 10^{-24}$ & 8.9 & $10^{6}$ & 200 & \\
\hline \multirow[t]{3}{*}{ M82 } & NFW & $4.0 \times 10^{-27}$ & 11 & $10^{6}$ & 0.2 & \multirow[t]{3}{*}{298.7} \\
\hline & Moore & $1.79 \times 10^{-27}$ & 13 & $10^{6}$ & 0.2 & \\
\hline & Burkert & $6.29 \times 10^{-27}$ & 9.0 & $10^{6}$ & 0.2 & \\
\hline
\end{tabular}
considered galaxy by assuming that the $f=1$, i.e., the full halo is composed of these clouds is shown in Fig. 1.

Table 1 Physical parameters of the considered nearby spiral galaxies

The central density $\rho_{c}$ (column 3) in units of $\mathrm{g} \mathrm{cm}^{-3}$, the core radius $r_{c}$ in kpc (column 4), the ratio of the central number density of the gas cloud $n_{\mathrm{cl}} \simeq 1 \times 10^{2} \mathrm{~cm}^{-3}$ to the number density of the IM in the particular galaxy (column 5), the dynamical mass (column 6), and the mass of each gas cloud (column 7), are given for Centaurus-A, M33, M81, and M82 galaxies 


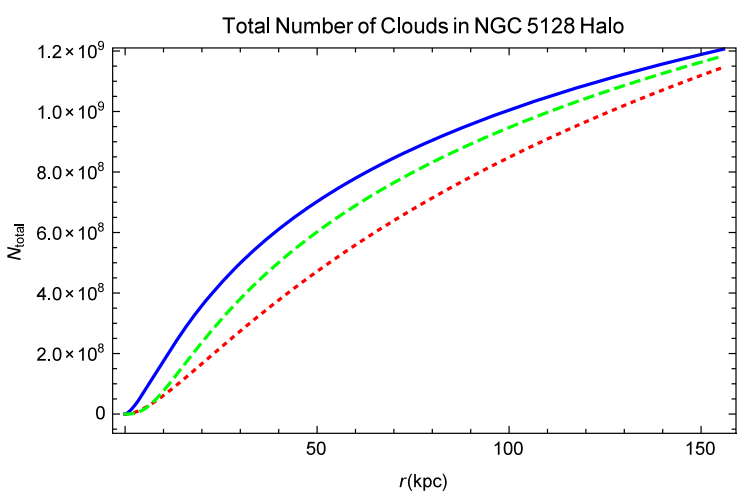

(a)

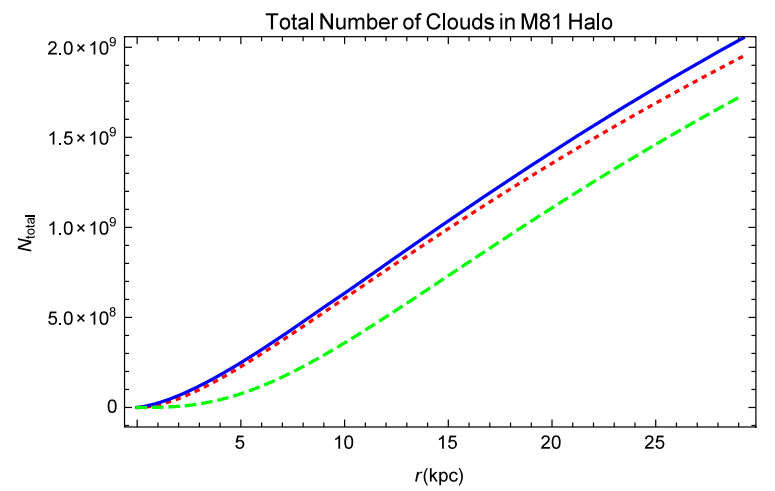

(c)

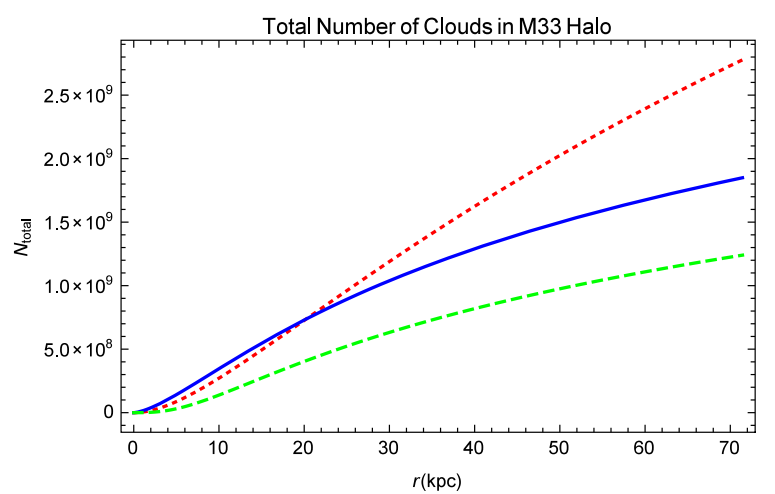

(b)

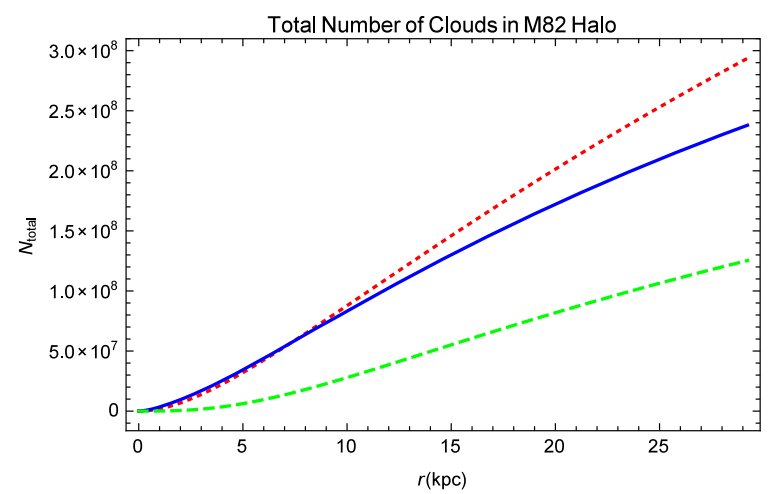

(d)

Fig. 1 The curves in the figure represent the total number of gas clouds in the galactic halo, with the assumption that the total halo of the galaxy is composed of these clouds, i.e., $f=1$. The red-dotted, blue-bold, and green-dashed represent $N_{\text {total }}$ for NFW, Moore and Burkert models. a Gives the total number of gas clouds in NGC 5128 within $150 \mathrm{kpc}$; b gives the total number of gas clouds in M33 within $70 \mathrm{kpc}$; $\mathbf{c}$ gives the total number of gas clouds in M81 within $25 \mathrm{kpc}$; and $\mathbf{d}$ gives the total number of gas clouds in M82 within $25 \mathrm{kpc}$

\section{Halo rotational velocity}

The rotational velocity of a galactic halo can be estimated by the relation,

$$
v_{\mathrm{rot}}=\left(\frac{\Delta T}{T_{\mathrm{CMB}}}\right)\left(\frac{c}{2 \sin i \tau_{\mathrm{eff}}}\right)
$$

where $c$ is the light speed, $i$ is the inclination angle of the rotational axis of the considered galaxy with respect to the line of sight, $\tau_{\text {eff }}=f S \bar{\tau}$ is the effective optical depth of the clouds ( $f$ is the fraction of the halo dark matter in the form of cold gas clouds and $S$ is the cloud filling factor and $\bar{\tau}$ is the average optical depth of the clouds), and $\Delta T$ is the measured temperature asymmetry by Planck data [1]. ${ }^{1}$ The dynamical mass is defined as,

$$
M_{\mathrm{dyn}}=\frac{v_{\mathrm{rot}}^{2} R_{\mathrm{Halo}}}{G},
$$

where $R_{\text {Halo }}$ is the radius of the galactic halo, and $G$ is the Newton's gravitational constant. Equations (13) and (14) yield

$$
M_{\text {dyn }}\left(\leq R_{\text {Halo }}\right)=\left(\frac{R_{\text {Halo }}}{G}\right)\left(\frac{\Delta T}{T_{\mathrm{CMB}}} \frac{c}{2 \sin i \tau_{\mathrm{eff}}}\right)^{2}
$$

\footnotetext{
${ }^{1}$ We have used the data published in the literature for the various galaxies $[11,12,16,17]$ in the $70 \mathrm{GHz}, 100 \mathrm{GHz}$, and 143 $\mathrm{GHz}$ bands at resolution corresponding to $N_{\text {side }}=2048$ in HEALPix scheme [15]. The temperature excesses are shown in Table 2 (column 4).
} 
Table 2 Estimated effective optical depth and halo rotational velocity of the considered galaxies

\begin{tabular}{|c|c|c|c|c|c|c|}
\hline Galaxies & $i$ & $\begin{array}{l}\text { Frequency } \\
(\mathrm{GHz})\end{array}$ & $\begin{array}{l}R_{\text {Halo }} \\
(\mathrm{kpc})\end{array}$ & $\begin{array}{l}\Delta T \\
(\mu \mathrm{K})\end{array}$ & $\begin{array}{l}\tau_{\text {eff }} \\
\left(10^{-3}\right)\end{array}$ & $\begin{array}{l}v_{\text {rot }} \\
\left(\mathrm{km} \mathrm{s}^{-1}\right)\end{array}$ \\
\hline Cen-A & $14.6^{0}$ & 143 & $\begin{array}{r}92 \\
171 \\
245 \\
92 \\
171 \\
245 \\
92 \\
171 \\
245\end{array}$ & $\begin{array}{l}70 \\
32 \\
12 \\
60 \\
42 \\
18 \\
58 \\
46 \\
19.5\end{array}$ & $\begin{array}{l}4.80 \\
4.43 \\
4.40 \\
4.46 \\
5.11 \\
5.35 \\
3.27 \\
4.00 \\
4.17\end{array}$ & $\begin{array}{l}89 \\
83 \\
74 \\
41 \\
53 \\
58 \\
15 \\
23 \\
24\end{array}$ \\
\hline M33 & $59^{0}$ & $\begin{array}{r}70 \\
100\end{array}$ & $\begin{array}{r}92 \\
171 \\
245 \\
92 \\
171 \\
245 \\
92 \\
171 \\
245\end{array}$ & $\begin{array}{l}70 \\
30 \\
14 \\
60 \\
42 \\
18 \\
58.5 \\
46 \\
19.5\end{array}$ & $\begin{array}{l}4.05 \\
3.78 \\
3.71 \\
3.61 \\
4.28 \\
4.48 \\
2.96 \\
3.25 \\
3.49\end{array}$ & $\begin{array}{r}150 \\
139 \\
125 \\
64 \\
90 \\
98 \\
30 \\
38 \\
41\end{array}$ \\
\hline M81 & $14^{0}$ & $\begin{array}{r}70 \\
100 \\
143\end{array}$ & $\begin{array}{l}15 \\
30 \\
60 \\
15 \\
30 \\
60 \\
15 \\
30 \\
60\end{array}$ & $\begin{array}{l}55.5 \\
65 \\
80 \\
40 \\
55 \\
75 \\
45 \\
50 \\
75\end{array}$ & $\begin{array}{l}1.10 \\
0.93 \\
0.99 \\
1.66 \\
1.55 \\
1.47 \\
2.64 \\
2.56 \\
2.56\end{array}$ & $\begin{array}{l}312 \\
265 \\
253 \\
366 \\
310 \\
281 \\
451 \\
422 \\
422\end{array}$ \\
\hline M82 & $26^{0}$ & $\begin{array}{r}70 \\
100\end{array}$ & $\begin{array}{l}15 \\
30 \\
60 \\
15 \\
30 \\
60 \\
15 \\
30 \\
60\end{array}$ & $\begin{array}{r}49.5 \\
42.5 \\
-16 \\
46 \\
48 \\
-18 \\
60 \\
60 \\
-12\end{array}$ & $\begin{array}{c}10.1 \\
13.1 \\
11.4 \\
9.68 \\
14.0 \\
12.1 \\
11.0 \\
15.6 \\
9.9\end{array}$ & $\begin{array}{r}286 \\
244 \\
-92 \\
275 \\
276 \\
-103 \\
345 \\
346 \\
-69\end{array}$ \\
\hline
\end{tabular}

The values for the estimated effective optical depth $\tau_{\text {eff }}$, using Eq. (15), and the corresponding halo rotational velocity $v_{\text {rot }}$ are shown in Table 2 . In the case of Cen-A $v_{\text {rot }}$ is in the range $15--90 \mathrm{~km} \mathrm{~s}^{-1}$, depending strongly on the considered Planck band but not varying much with the value of $R_{\text {Halo }}$. In the case of the M33 galaxy, $v_{\text {rot }}$ is between 30 and $150 \mathrm{~km} \mathrm{~s}^{-1}$ and depends strongly on the considered frequency band. For the M81 galaxy $v_{\text {rot }}$ turns out to be in the range $250--450 \mathrm{~km} \mathrm{~s}^{-1}$; while for M82, it is between -100 and $345 \mathrm{~km} \mathrm{~s}^{-1}$. In particular, in the case of M82, the halo rotation velocity (which, in our model, is assumed to be fixed and equal to that observed for the galactic disk, that is certainly a simplified assumption) seems to change sign in the outer galactic region, outside about 30-60 kpc from the galactic center. Actually, the signs of a complex behavior of the M82 dynamics were already present in the literature and interpreted as possibly resulting from the interaction of M82 with the M81 and/or NGC 3077 galaxies in the past [17].

For the considered galaxies, we give the inclination angle $i$ of the rotation axis with respect to the line of sight (column 2), the central frequency of the considered Planck band (column 3), the corresponding radius of the halo $R_{\text {Halo }}$ (column 4 ) within which the temperature asymmetry $\Delta T$ (column 5 ) is detected. In the sixth column, we give the estimated cloud effective optical depth $\tau_{\text {eff }}$; while in column 7 , the corresponding value of the halo rotational velocity $v_{\text {rot }}$ is shown.

\section{Conclusion}

While it is clear that spiral galaxy disks rotate, we have no idea how galactic halos rotate. To determine what the nature of the gas clouds is, we confront the model with the data, for the observed temperature asymmetry in the Planck data towards some nearby galaxies. 
In spite of the oversimplified toy model adopted to describe the gas clouds, assumed to be isothermal and at the CMB temperature, self-gravitating, entirely composed of $\mathrm{H}_{2}$ molecules and rotating around the same rotation axis as the galactic disk, the obtained values of $v_{\text {rot }}$ (see Table 2) appear to be not very far from the rotation speed of the galaxy stellar disks. From Table 1, we see that the mass of the clouds (see Table 1 Column 7) does not change much with the change in the galaxy; it is approximately equal for NGC 5128, M33, M81, and M82. Thus, the density of the IM does not affect the mass of the cloud, as had been expected. The clouds are thought to be optically thick, i.e., $\bar{\tau}=1$, This is certainly a simplifying assumption since a correct calculation of $\bar{\tau}$ would involve summing up the integrated spectral contribution, of the individual line transitions, in a certain Planck band. The number of line transitions in the Planck spectral bands is very high, and depends on the chemical species present in each cloud. However, Kaifu et al. [19] performed a spectral line survey toward the cold, dark cloud TMC-1 (cyanopolyyne peak) in the frequency range between 8.8 and $50.0 \mathrm{GHz}$ (which overlaps with the lowest frequency Planck spectral bands) by the $45-\mathrm{m}$ radio telescope of Nobeyama Radio Observatory, detecting 414 lines of 38 different molecular species. These lines appear spectrally rather dense and, in the innermost region of the assumed halo, the clouds may form by the fragmentation, where the gas density is higher and the molecule collisions are more frequent; it does not appear that the clouds are nearly optically thick, while the outer regions of the clouds should be, clearly, optically thin [27]. Since the clouds are at the CMB temperature and are neither absorbing nor emitting more radiations. It might be possible that their luminosity must be equal to the CMB luminosity. It means that their luminosity is equal to the Doppler shift observed. Further work is needed to relax these simplifying assumptions.

We have only looked at the possibility of these cold gas clouds made of molecular hydrogen. We have not looked at the possibility of these clouds contaminated with other interstellar matter like interstellar dust. One can also try to model this possibility. These clouds might be contaminated with more or less interstellar dust or other heavier elements in the IM, but we have not discussed the contamination of radiation by matter at high temperature. This possibility needs to be eliminated, because the effect is probably negligible, but cannot be neglected without checking (Work on this issue is in progress.). One also needs to investigate how the clouds would have evolved over billions of years, from the time when the CMB temperature was higher than it is now to the present value. Precisely, how did the clouds lose energy over this period?

Acknowledgements We acknowledge the use of Planck's data in the Legacy Archive for Microwave Background Data Analysis (LAMBDA) and HEALPix [15] package. We would also like to acknowledge the project Inter-Asia 2018, by the collaboration of National University of Sciences and Technology, Islamabad, Pakistan and University of Salento, Lecce, Italy, and INFN which helped us to interact and work on this project. NT is grateful for the hospitality to the ISUFI college in campus, Eckotecne, of University of Salento, Lecce, Italy. FDP and AAN acknowledge the TAsP and Euclid INFN projects.

Open Access This article is distributed under the terms of the Creative Commons Attribution 4.0 International License (http:// creativecommons.org/licenses/by/4.0/), which permits unrestricted use, distribution, and reproduction in any medium, provided you give appropriate credit to the original author(s) and the source, provide a link to the Creative Commons license, and indicate if changes were made.

\section{References}

1. Ade, P.A.R.; et al. (Planck Collaboration): Planck 2015 results I. Overview of products and scientific results. Astron. Astrophys. 514, A1 (2016)

2. Bonnor, W.B.: Boyle's law and gravitational instability. Mon. Not. R. Astron. Soc. 116, 351 (1956)

3. Burkert, A., Silk, J.: On the structure and nature of dark matter halos, 375 (1999)

4. Cen, R.; Ostriker, J.P.: Where are the Baryons? II. Feedback effects. Astrophys. J. 650, 560 (2006)

5. Corbelli, E.: Dark matter and visible baryons in M33. Mon. Not. R. Astron. Soc. 342, 199 (2003)

6. Courteau, S.; Widrow, L.M.; McDonald, M.; Guhathakurta, P.; Gilbert, K.M.; Zhu, Y.; Beaton, R.L.; Majewski, S.R.: The luminosity profile and structural parameters of the andromeda galaxy. Astrophys. J. 739, 20 (2011)

7. De Paolis, F.; Ingrosso, G.; Jetzer, P.; Roncadelli, M.: A scenario for a baryonic dark halo. Astron. Astrophys. 299, 647 (1995a)

8. De Paolis, F.; Ingrosso, G.; Jetzer, P.; Qadir, A.; Roncadelli, M.: Observing molecular hydrogen clouds and dark massive objects in galactic halos. Astron. Astrophys. J. 299, 647 (1995b)

9. De Paolis, F.; Ingrosso, G.; Jetzer, P.; Roncadelli, M.: Halo dark clusters of brown dwarfs and molecular clouds. Astrophys. J. 500, $59(1998)$

10. De Paolis, F.; Gurzadyan, V.G.; Nucita, A.A.; Ingrosso, G.; Kashin, A.; Khachatryan, H.; Mirzoyan, S.; Poghosian, E.; Jetzer, P.; Qadir, A.: Planck confirmation of the disk and halo rotation of M 31. Astron. Astrophys. 565, L3 (2014)

11. De Paolis, F.; Gurzadyan, V.G.; Nucita, A.A.; Ingrosso, G.; Kashin, A.L.; Khachatryan, H.G.; Mirzoyan, S.; Yegorian, G.; Jetzer, P.; Qadir, A.; Vetrugno, D.: Planck revealed bulk motion of Centaurus-A lobes. Astron. Astrophys. 580, L8 (2015)

12. De Paolis, F.; Gurzadyan, V.G.; Nucita, A.A.; Chemin, L.; Qadir, A.; Kashin, A.L.; Khachatryan, H.G.; Sargsyan, S.; Yegorian, G.; Ingrosso, G.; Jetzer, P.; Vetrugno, D.: Triangulum galaxy viewed by Planck. Astron. Astrophys. 593, A57 (2016) 
13. Ebert, R.: Über die Verdichtung von H I-Gebieten. Mit 5 Textabbildungen. Zeitschrift für Astrophysik 37, 217 (1955)

14. Fraser-McKelvie, A.; Pimbblet, K.A.; Lazendic, J.S.: An estimate of the electron density in filaments of galaxies at $\mathrm{z} 0.1$. Mon Not. R. Astron. Soc. 415, 1961 (2011)

15. Gorski, K.M.; Hivon, E.; Banday, A.J.; Wandelt, B.D.; Hansen, F.K.; Reinecke, M.; Bartelmann, M.: HEALPix: a framework for high-resolution discretization and fast analysis of data distributed on the sphere. Astrophys. J. 622, 759 (2005)

16. Gurzadyan, V.; De Paolis, F.; Nucita, A.A.; Ingrosso, G.; Kashin, A.L.; Khachatryan, H.G.; Sargsyan, S.; Yegorian, G.; Jetzer, P.; Qadir, A.; Vetrugno, D.: Planck view of the M 82 galaxy. Astron. Astrophys. 582, A77 (2015)

17. Gurzadyan, V.G.; De Paolis, F.; Nucita, A.A.; Kashin, A.L.; Amekhyan, A.; Sargsyan, S.; Yegorian, G.; Qadir, A.; Ingrosso, G.; Jetzer, P.; Vetrugno, D.: Messier 81's Planck view versus its halo mapping. Astron. Astrophys. 609, A131 (2018)

18. Johnny, P.G.; Paul, M.; Todd, A.T.: Measurement of the mass and stellar population distribution in M82 with the LBT. Astrophys. J. 757, 1 (2012)

19. Kaifu, N.; Ohishi, M.; Kawaguchi, K.; Saito, S.; Yamamoto, S.; Miyaji, T.; Miyazawa, K.; Ishikawa, S.; Noumaru, C.; Harasawa, S.; Okuda, M.; Suzuki, H.: A 8.8-50 GHz complete spectral line survey toward TMC-1 I. Survey data. Publ. Astron. Soc. Jpn. 56, 69 (2004)

20. Mathur, S.; Sivakoff, G.R.; Williams, R.J.; Nicastro, F.: On the nature of the $z=0$ X-ray absorbers: I. Clues from an external group. Astrophys. Space Sci. 315, 93 (2008)

21. Mathieu, A., Dejonghe, H., Hui, X.: Probing the halo of Centaurus A: a merger dynamical model for the PN population 309, $30(1996)$

22. Moore, B.; Quinn, T.; Governato, F.; Stadel, J.; Lake, G.: Cold collapse and the core catastrophe. Mon. Not. R. Astron. Soc. 310, 1147 (1999)

23. Navarro, J.F.; Frenk, C.S.; White, S.D.M.: The structure of cold dark matter Halos. Astrophys. J. 462, 563 (1996)

24. Oehm, W.; Thies, I.; Kroupa, P.: Constraints on the dynamical evolution of the galaxy group M81. Mon. Not. R. Astron. Soc. 467, 273 (2017)

25. Revaz, Y.; Pfenniger, D.; Combes, F.; Bournaud, F.: Simulations of galactic disks including a dark baryonic component. Astron. Astrophys. 501, 171 (2009)

26. Tahir, N., De Paolis, F., Qadir, A., Nucita, A.A.: Constraining baryons in the M31 galactic halo using Planck data. Int. J. Modern Phys. D (2018) (submitted)

27. Wardle, M.; Walker, M.: Thermal stability of cold clouds in galaxy halos. Astrophys. J. 527, L109 (1999)

Publisher's Note Springer Nature remains neutral with regard to jurisdictional claims in published maps and institutional affiliations. 